\title{
THERMAL STRUCTURE OF A PROTOSTELLAR ENVELOPE
}

\author{
Ya.N. Pavlyuchenkov ${ }^{1 *}$ A.G. Zhilkin ${ }^{1,2}$, E.I. Vorobyov ${ }^{3,4}$, \\ A.M. Fateeva ${ }^{1}$ \\ ${ }^{1}$ Institute of Astronomy, Russian Academy of Sciences, Pyatnitskaya st. \\ 48, Moscow, 119017 Russia \\ ${ }^{2}$ Chelyabinsk State University, Chelyabinsk, Russia \\ ${ }^{3}$ Research Institute of Physics, Southern Federal University, \\ Rostov-on-Don 344090, Russia \\ ${ }^{4}$ Institute of Astrophysics, University of Vienna, Vienna, Austria
}

\begin{abstract}
A numerical hydrodynamical model for the evolution of spherically symmetric collapsing clouds, designed for the calculation of the thermal structure of these objects in both the prestellar and protostellar stages of their evolution, is presented. Distinctive features of the model include the possibility of independently describing the temperatures of the gas and dust, which is extremely important when calculating the thermal structure of prestellar and protostellar clouds, and the account of the radiation flux from the central protostar. This model is used to compare the theoretical density and temperature distributions with observations for nearby sites of star formation obtained with the Herschel Space Observatory. Application of the diffusion approximation with a flux limiter describes well the radial density and temperature distributions in protostellar clouds. However, significant differences between the model and observational density profiles were found for prestellar stages, suggesting the presence of appreciable deviations from equilibrium in the prestellar clouds. An approximate method for calculating the thermal structure of a cloud based on the adaptive $\tau$-approximation is presented. Application of the $\tau$-approximation yields good agreement with the diffusion approximation for the prestellar phase, but produces appreciable discrepancies for the protostellar phase, when the thermal structure of the accreting envelope is determined by the radiation of the protostar.
\end{abstract}

\section{Introduction}

A model for calculating the thermal structure of a collapsing protostellar cloud was presented in $[1,2]$. This model is fast enough for use in hydrodynamic calculations,

*E-mail: pavyar@inasan.ru 
and is also sufficiently accurate to use the modeling results to calculate theoretical spectra that can be compared with observations. The key feature of the model is separate treatment of dust and gas temperatures, since these two temperatures may differ significantly in early phases of the evolution of the clouds, and also in their outer regions. Different heating and cooling mechanisms are considered for the gas and dust, while they exchange energy through collisions. Since the main source of heating (cooling) of the dust is the absorption (emission) of radiation, the method used to calculate the radiation transfer is an important component of the model.

The basic idea of the radiative transfer computation method is to divide the overall frequency range into low-frequency (infrared) and high frequency (ultraviolet) parts. The high-frequency part is diluted by interstellar radiation. The intrinsic radiation of the dust can be neglected in this part of the spectrum, and the absorption (and scattering) of external radiation alone can be considered. In the low-frequency part of the spectrum, it is necessary to consider both absorption and thermal emission of the ambient medium. This division of the spectral range into two parts enables the use of different appropriate approximate methods in each. Modeling the UV part of the spectrum reduces to calculating the mean intensity of the UV radiation via direct integration of the radiative transfer equation along selected directions. The calculation of the intensity of the IR radiation is based on solving a system of moment equations that represent the diffusion approximation. Thus, the model contains four interacting components: gas, dust, and the IR and UV radiation.

An axially symmetric realization of this thermal model was used to calculate the early evolution of a contracting, magnetized protostellar cloud [1]. Modeling of later stages, up to the formation of the first hydrostatic core, was performed in a spherically symmetric approximation in [2]. In our present study, we describe a modification of the model designed for the calculation of the accretion phase in the evolution of a protostellar cloud following the formation of the protostar. We present the results of our calculations of the structure of a spherically symmetric, contracting protostellar cloud and accreting envelope. To simplify the computation of the thermal structure, we propose the adaptive $\tau$-approximation method and compare it with the original method. We also compare the model and observed distributions of the density and temperature in prestellar and protostellar cores of molecular clouds.

\section{MODIFICATION OF THE MODEL}

Pavlyuchenkov and Zhilkin [2] investigated the thermal evolution of a protostellar cloud up to the formation of the first hydrostatic core. Evolution of this core subsequently leads to the evaporation of dust, dissociation of hydrogen, and, consequently, to the collapse of the first core and the formation of a second hydrostatic core, which is essentially the protostar [3-5]. Simulation of these stages requires that additional physical processes be taken into account in the model for the protostellar cloud. The timescales for these stages are extremely short compared to the dynamical timescale of the accreting envelope. This makes hydrostatic modeling of the core and accreting envelope using a single model extremely difficult. Moreover, multi-dimensional models must be used in studies of hydrostatic cores, since their formation and evolution are closely associated with the formation of accretion 
(protoplanetary) disks and outflows from them. However, in addition to modeling the evolution of a protostar and its surroundings (the protostellar disk), studies of the structure of the extended accreting envelope (including its thermal structure) are of interest. Accretion from the envelope supplies matter to the protostar and protostellar disk, determining their evolution. In turn, the observational identification of protostellar accreting cores depends on assumptions about their thermal structure, which requires appropriate models.

Historically, the simplest models for prestellar and protostellar envelopes were based on semianalytical self-similar solutions of the hydrodynamical equations in isothermal or polytropic approximations $[7,8]$, with possible modifications to take into account rotation in the cloud [9], as well as numerical hydrodynamical modeling taking into account the finite size of the cloud [10-12]. Later, numerical models were used to consider the nonisothermal structure of the envelopes more accurately, via more accurate solution of the transfer equation taking into account the frequency dependence of the absorption coefficient [13], and applying the diffusion approximation with a flux limiter and either mean [14] or frequency-dependent [15] opacities. Modeling of protostars and protostellar envelopes has been carried out by a number of research groups (see, e.g., [6]). However, most models still assume that the temperatures of the gas and dust are equal, since frequent collisions should establish thermal equilibrium between these components. As is noted above, however, this assumption may not be valid at some stages of the evolution. In some models, the dust and gas were treated separately by postprocessing the gas density distribution in the envelope using Monte-Carlo methods for the radiative transfer [16]. Finally, most studies have focused on early stages in the evolution of the first hydrostatic core and protostar [17]. Here, we consider a collapsing cloud and describe a modification of our model [2] designed for the calculation of the thermal structure of an accreting envelope.

The model of [2] is based on a numerical solution of the hydrodynamical and radiative transfer equations. The continuity equation, equation of motion, and the equation describing the variations of the thermal energy of the gas $E_{\mathrm{g}}$ have the form

$$
\begin{array}{r}
\frac{\partial \rho}{\partial t}+\nabla \cdot(\rho \mathbf{v})=0 \\
\frac{\partial \mathbf{v}}{\partial t}+(\mathbf{v} \cdot \nabla) \mathbf{v}=-\frac{\nabla p}{\rho}+\mathbf{g}, \\
\frac{\partial E_{\mathrm{g}}}{\partial t}+\nabla \cdot\left(E_{\mathrm{g}} \mathbf{v}\right)+p \nabla \cdot \mathbf{v}=\Gamma_{\mathrm{g}}-\Lambda_{\mathrm{g}}-\Lambda_{\mathrm{gd}}
\end{array}
$$

When the velocity of the medium is low, the equations for the thermal energy of the dust $E_{\mathrm{d}}$ and the energy of the IR radiation $E_{\mathrm{r}}$ have the form

$$
\begin{array}{r}
\frac{\partial E_{\mathrm{d}}}{\partial t}=-\sigma_{P}\left(a T_{\mathrm{d}}^{4}-E_{\mathrm{r}}\right)+\Lambda_{\mathrm{gd}}+S \\
\frac{\partial E_{\mathrm{r}}}{\partial t}=\nabla \cdot\left(\frac{1}{\sigma_{R}} \nabla E_{\mathrm{r}}\right)+\sigma_{P}\left(a T_{\mathrm{d}}^{4}-E_{\mathrm{r}}\right) .
\end{array}
$$

In these equations, $\rho$ is the density, $\mathbf{v}$ the velocity, $p$ the pressure, $\mathbf{g}$ the gravitational acceleration, $\Gamma_{\mathrm{g}}$ the gas heating function, $\Lambda_{\mathrm{g}}$ the gas cooling function, $\Lambda_{\mathrm{gd}}$ 
the rate of exchange of thermal energy between the gas and dust, $S$ the rate of dust heating by UV radiation, $T_{\mathrm{d}}$ the dust temperature, $\sigma_{R}$ and $\sigma_{P}$ coefficients that depend on the opacity of the medium, and a the radiative constant. A detailed description of the system of equation and the solution method is given in [2]. Here, we will discuss our modification of this model.

The modification was carried out in the framework of the well-known "sink cell" formalism (see, e.g., [18]). In this approach, the central region of the cloud (the sink cell) is excluded from the simulation when a high density is achieved there. This makes it possible to exclude from the modeling the rapid evolution of this region with numerous physical processes leading to the formation of the central star. However, the influence of this region on the surrounding cloud is taken into account, as well as the influence of the cloud on the inner region. This interrelation has both a dynamical and thermal nature. Thus, the modification of the model affects the hydrodynamical method and the method used to compute the thermal structure. The modification of the hydrodynamical method depends on the type of method used (Eulerian, Lagrangian, smoothed particle hydrodynamics method, etc.).

We will now describe the changes in the one-dimensional Lagrangian method used. Let $r_{\text {cell }}$ be the radius of the inner sink cell. As the cloud contracts, the cells of the computational domain become denser and move toward the center. We will suppose that a cell is excluded from the calculations (it is absorbed by the sink cell) as soon as the right-hand border of the cell crosses the radius $r_{\text {cell }}$. The next cell then becomes the boundary cell, and the cells are renumbered such that the new boundary cell is assigned the subscript " 1 ". Thus, the left-hand boundary of the new boundary cell $r_{1}$ will be located to the left of $r_{\text {cell }}$, while its right-hand boundary $r_{2}$ is located to the right of $r_{\text {cell }}$. We assume that the pressure gradient vanishes at the left border $\left(r_{1}\right)$ :

$$
\left.\frac{d p}{d r}\right|_{r_{1}}=0
$$

This condition means that the matter flows freely (by inertia) into the inner zone under the action of gravity of the sink cell alone.

The modification of the method used to compute the thermal structure involves two operations: taking into account the sink cell and modifying the diffusion operator. Taking into account the sink cell reduces to formulating a new boundary condition at the inner boundary of the computational domain $r_{1}$, where the IR flux from the sink cell $L_{\text {cell }}$ is determined by the sum of the photospheric and accretion luminosities of the young star:

$$
L_{\text {cell }}=L_{\text {star }}+L_{\text {acc }} .
$$

We calculated the photospheric luminosity using the stellar model of $[19,20]$, where the luminosity $L_{\text {star }}$ and the radius of the young star $R_{\text {star }}$ are computed as functions of the instantaneous $M_{\text {star }}$ and age $t_{\text {star }}$. Assuming equipartition between the thermal and kinetic (mechanical) energy of the accreting matter 1 , the accretion luminosity is given by

\footnotetext{
${ }^{1}$ The mechanical energy does not contribute to the radiation and is mostly carried away together with polar jets and outflows of matter.
} 


$$
L_{\mathrm{acc}}=\frac{1}{2} \frac{G \dot{M} M_{\mathrm{star}}}{R_{\mathrm{star}}}
$$

where

$$
\dot{M}=4 \pi r_{1}^{2} \rho_{1} v_{1}
$$

is the accretion rate through the inner boundary of the computational domain. Here, $\rho_{1}$ is the density in the first (boundary) cell, and $v_{1}$ is the radial velocity at the left-hand border of the first cell.

When calculating the accretion luminosity using these formulas, we assumed that all the matter crossing the boundary of the computational domain is instantaneously accreted onto the star, and the all thermal energy of the accreted matter is converted into IR radiation; this corresponds to the so-called cold accretion limit, and is valid if the accretion rate onto the protostar is not too high, $\dot{M} \lesssim 10^{-5} M_{\odot} /$ yr [21]. Since the coordinate in the Lagrangian method is the mass inside the corresponding radius, the mass inside the absorbing zone can be calculated directly from the coordinates of the boundary cell:

$$
M_{\mathrm{star}}=4 \pi q_{1}
$$

where $q_{1}$ is mass (Lagrangian) coordinate.

Finite-difference approximation of the diffusion operator in the sphericallysymmetric case

$$
\begin{aligned}
& \nabla \cdot\left(\frac{1}{\sigma} \nabla E\right)=\frac{1}{r^{2}} \frac{\partial}{\partial r}\left(\frac{r^{2}}{\sigma} \frac{\partial E}{\partial r}\right) \text { has the form 2: } \\
& \hat{\Lambda} E=\frac{1}{R_{i+1 / 2}^{2} \Delta r_{i+1 / 2}}\left(\frac{R_{i+1}^{2}}{\sigma_{i+1}} \frac{E_{i+3 / 2}-E_{i+1 / 2}}{\Delta r_{i+1}}-\frac{R_{i}^{2}}{\sigma_{i}} \frac{E_{i+1 / 2}-E_{i-1 / 2}}{\Delta r_{i}}\right),
\end{aligned}
$$

where

$$
\begin{array}{r}
R_{i+1 / 2}^{2}=\frac{1}{3}\left(r_{i}^{2}+r_{i} r_{i+1}+r_{i+1}^{2}\right), \\
R_{i}^{2}=r_{i-1 / 2} r_{i+1 / 2}, \\
\Delta r_{i}=r_{i+1 / 2}-r_{i-1 / 2}, \\
\Delta r_{i+1 / 2}=r_{i+1}-r_{i} .
\end{array}
$$

Taking into account the new boundary condition, the finite-difference approximation of this operator for the inner boundary of the cell takes the form

$$
\hat{\Lambda} E=\frac{1}{R_{1 / 2}^{2} \Delta r_{1 / 2}}\left(\frac{R_{1}^{2}}{\sigma_{1}} \frac{E_{3 / 2}-E_{1 / 2}}{\Delta r_{1}}+\frac{L_{\text {cell }}}{4 \pi}\right),
$$

We introduce the sink cell when the central density becomes $n\left(\mathrm{H}_{2}\right)=10^{14} \mathrm{~cm}^{-3}$. The central temperature at this time is close to $1000 \mathrm{~K}$. The radius of the sink cell

\footnotetext{
${ }^{2}$ Note that there is an error in Eq. (38) in [2].
} 
we used is $r_{\text {cell }}=50 \mathrm{AU}$. This is appreciably larger than the radius of the first hydrostatic core, but appreciably smaller than the extent of the accreting envelope. This choice ensures that the sink cell is larger than the first hydrostatic core. Otherwise, the formation of a quasi-hydrostatic structure at the inner boundary of the computational mesh would hinder the accretion of matter onto the sink cell. When the sink cell is introduced, all the grid cells inside this zone are eliminated from further consideration. The time when the sink cell is introduced is also taken to be the time when the star forms, when we start to compute the photospheric and accretion luminosity.

Other numerical criteria for the formation of the protostar are also possible. In particular, it was found in [4] that the maximum mass of the first hydrostatic core (i.e., the mass at the beginning of the dissociation of molecular hydrogen and the subsequent formation of the protostar) was close to $0.05 M_{\odot}$, independent of the initial mass of the cloud. This can be used to define the moment of the protostar's formation. However, this value was obtained for nonrotating, spherically symmetric clouds. Taking into account the angular momentum of the cloud could lead to a reassessment of the maximum mass of the first hydrostatic core [22].

The Eddington approximation was applied in the equation for the energy of the IR radiation in [2]. This approximation assumes that the radiation field is close to isotropic, making it possible to express the radiation pressure tensor $\hat{P}_{\mathrm{r}}$ in terms of the radiation energy $E_{\mathrm{r}}$ in the form $\hat{P}_{\mathrm{r}}=\frac{1}{3} \hat{I} E_{\mathrm{r}}$, where $\hat{I}$ is the unit tensor. The diffusion coefficient in (5) is then

$$
D_{\mathrm{E}}=1 / \sigma_{R}=\frac{c}{3 \rho_{\mathrm{d}} \kappa_{R}},
$$

where $\rho_{\mathrm{d}}$ is the dust density, $\kappa_{R}$ the Rosseland mean opacity, and $c$ the speed of light. However, the Eddington approximation yields large errors when the radiation propagates from a localized source in an optically thin medium (in the so-called streaming regime). The accretion stage in the evolution of a protostellar cloud proceeds in precisely this regime, with the protostar playing the role of the localized source and the accreting envelope being optically thin in the IR. Therefore, in place of the diffusion coefficient in the Eddington approximation, we used a flux limiter (so-called Flux Limited Diffusion, FLD) [23].

If the frequency-averaged scattering coefficient is substantially lower than the absorption coefficient (as is true in the IR), the diffusion coefficient is given by

$$
D_{\mathrm{FLD}}=\lambda \frac{c}{\rho_{\mathrm{d}} \kappa_{P}} \frac{a T_{\mathrm{d}}^{4}}{E_{\mathrm{r}}}
$$

where

$$
\lambda=\frac{2+f}{6+3 f+f^{2}}, \quad \mathbf{f}=-\frac{\nabla E_{\mathrm{r}}}{\rho_{\mathrm{d}} \kappa_{P} a T_{\mathrm{d}}^{4}},
$$

where $\kappa_{P}$ is the Planck mean absorption coefficient. In the iterative scheme used to derive $T_{\mathrm{d}}^{n+1}$ and $E_{\mathrm{r}}^{n+1}$, the values of $D_{\mathrm{FLD}}$ were computed using the dust temperature $T_{\mathrm{d}}^{n} \mathrm{~d}$ and the energy of the IR radiation $E_{\mathrm{r}}^{n}$ from the previous time step. The FLD approximation operates well in both the streaming regime and the optically thick regime, when $D_{\mathrm{FLD}} \approx D_{\mathrm{E}}$. Thus, the evolution of the accreting envelope and the preceding stage of the formation of the hydrostatic core should both be computed correctly in the FLD approximation. 

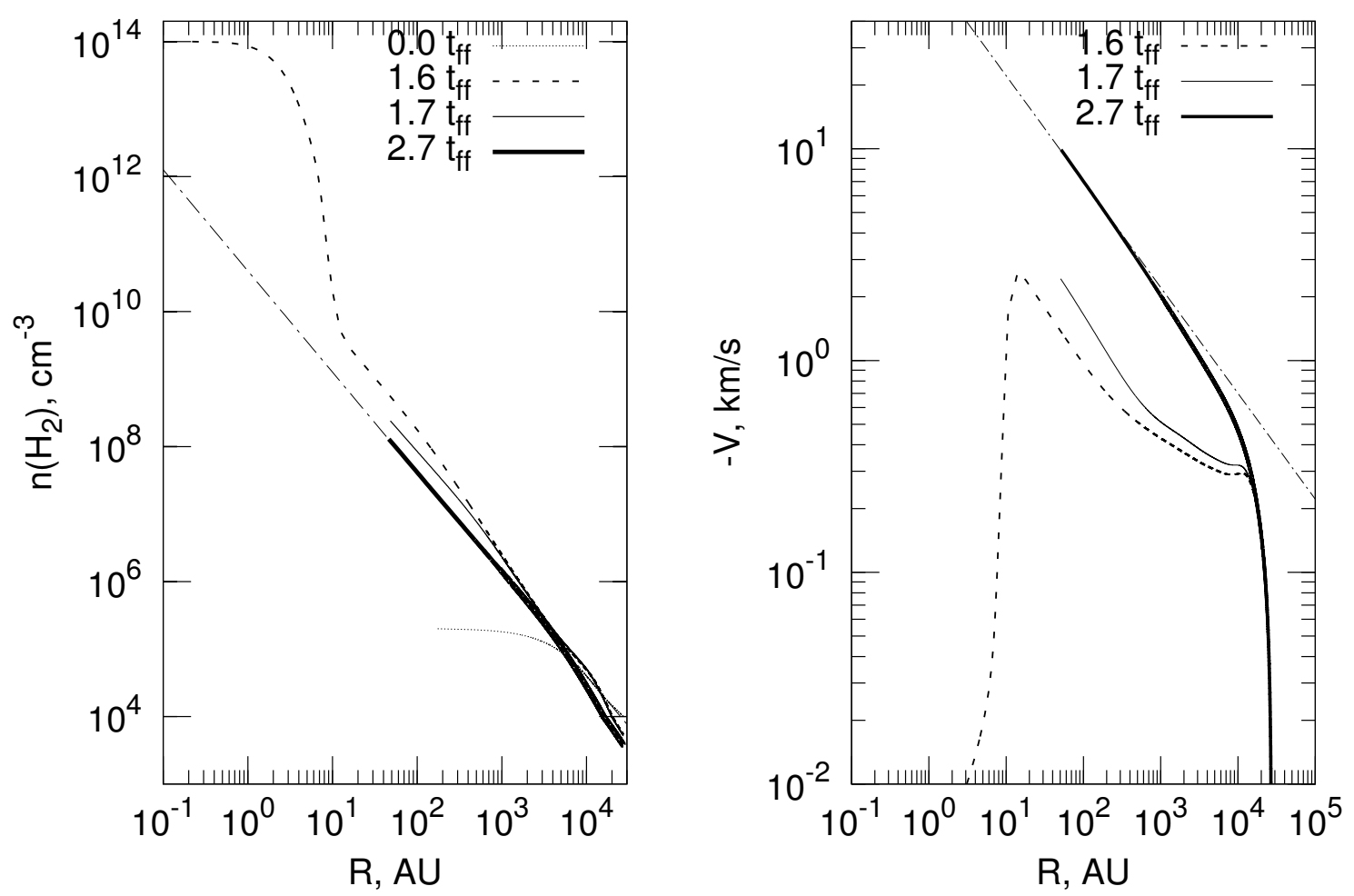

Figure 1: Distributions of the hydrogen number density (left) and the velocity (right) in the protostellar cloud at various times during its evolution. The dash.dot lines show the functions $n \propto r^{-3 / 2}$ and $v \propto r^{-1 / 2}$, whose indices are defined by the self-similar solution in the case of spherically symmetric accretion.

\section{EVOLUTION OF THE CLOUD WITH TRAN- SITION TO THE ACCRETION PHASE}

Figures 1 and 2 present the results of our modeling of the evolution of a protostellar cloud with the initial conditions taken from the model described in [2]. The distributions of the hydrogen number density $n\left(\mathrm{H}_{2}\right)$, velocity $v$, and the temperatures of the gas $\left(T_{\mathrm{g}}\right)$, dust $\left(T_{\mathrm{d}}\right.$, and IR radiation $\left(T_{\mathrm{r}}\right)$ are shown for four times. Let us describe the main features of these distributions for each of these times.

1. $t=0.0 t_{\mathrm{ff}}$. The initial protostellar cloud with parameters identical to those described in [2]. The distributions of the density and temperature were obtained for a cloud with central density $n\left(\mathrm{H}_{2}\right)=10^{5} \mathrm{~cm}^{-3}$ in hydrostatic and thermal equilibrium. The cloud was irradiated by external blackbody radiation with the temperature $10^{4} \mathrm{~K}$ and dilution $10^{-14}$. To initiate the contraction, the density in the hydrostatic cloud was increased by a factor of two. The temperature of the dust and gas in the initial configuration are different throughout volume, due to the relatively low density of the medium. The thermal structure is governed totally by the external radiation.

2. $t=1.6 t_{\mathrm{ff}}$. The first hydrostatic core. As a result of the cloud compression, the central hydrogen number density has reached $10^{14} \mathrm{~cm}^{-3}$, and the temperature $1000 \mathrm{~K}$. While the temperatures of the gas and dust in the inner regions 

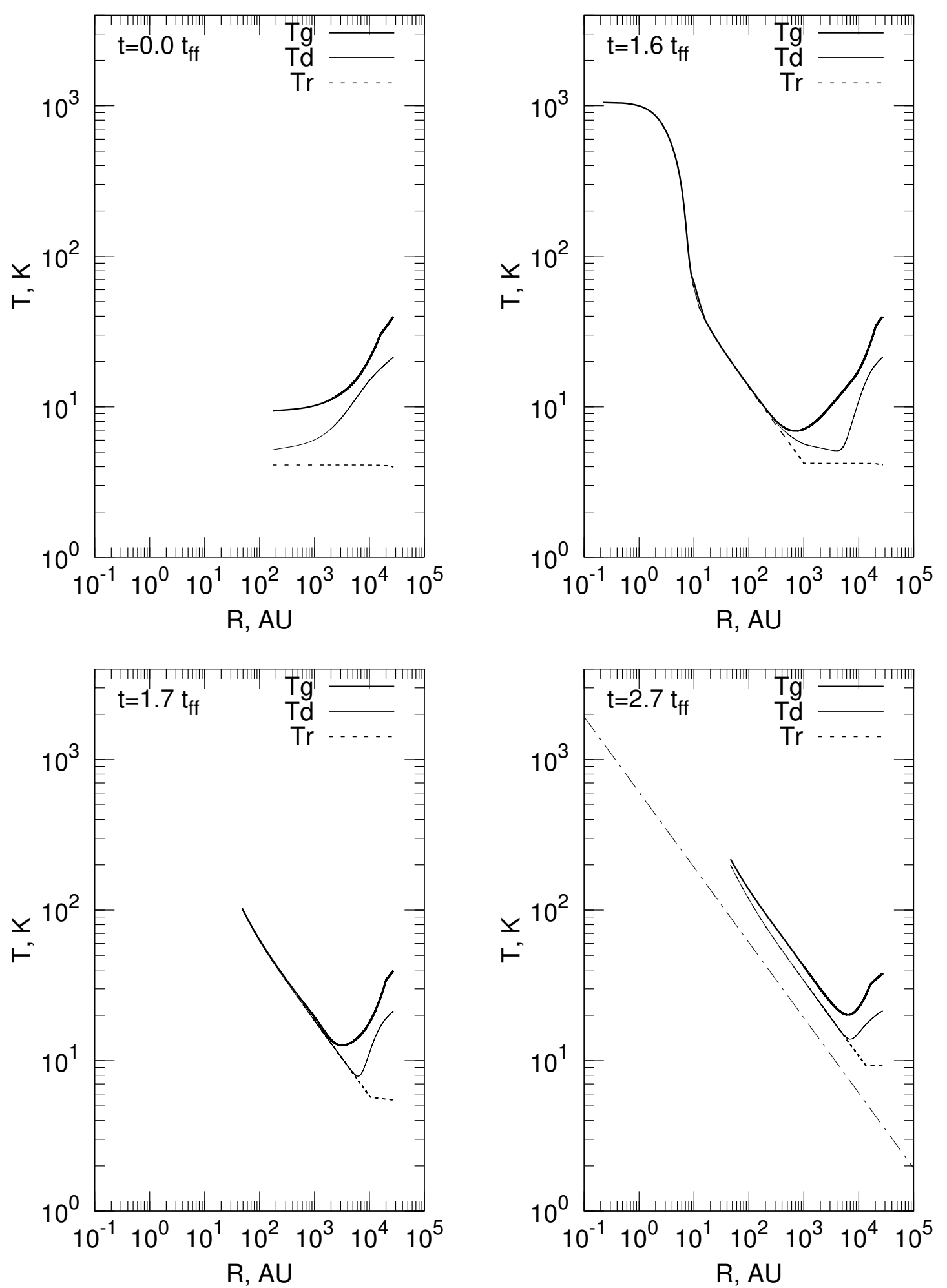

Figure 2: Distributions of the gas $\left(T_{\mathrm{g}}\right)$, dust $\left(T_{\mathrm{d}}\right)$, and radiation $\left(T_{\mathrm{r}}\right)$ temperatures at various times during the evolution of a protostellar cloud. The dash-dot line corresponds to the temperature distribution of the stellar radiation in the absence of absorption. 
$(r<300 \mathrm{AU})$ are equal, they remain different in the outer parts of the cloud. The temperature of the IR radiation is close to the temperature of the gas and dust inside $r<300 \mathrm{AU}$, indicating that this region is opaque to its own thermal radiation. The quasi-hydrostatic equilibrium of the inner region can be inferred from the velocity profile: the contraction rate is close to zero at $r<10 \mathrm{AU}$. This epoch corresponds to the time when the sink cell was introduced.

3. $t=1.7 t_{\mathrm{ff}}$. The time $0.1 t_{\mathrm{ff}}$ after the formation of the protostar and introduction of the sink cell. The temperatures of the gas, dust, and radiation are equal within $r<1000 \mathrm{AU}$, and reach $100 \mathrm{~K}$ at the inner boundary. At the same time, the temperatures in the envelope are close to those in the prestellar phase of the evolution. The hydrogen number density and gas velocity at the inner boundary are $10^{8} \mathrm{~cm}^{-3}$ and $2 \mathrm{~km} / \mathrm{s}$.

4. $t=2.7 t_{\mathrm{ff}}$. The protostellar phase, corresponding to a developed accretion regime. At this time, the mass of the star is $3 M_{\odot}$, its age is $90000 \mathrm{yr}$, its radius $11 R_{\odot}$, the accretion rate $3.6 \times 10^{-5} M_{\odot} / \mathrm{yr}$, the photospheric luminosity $64 L_{\odot}$, and the accretion luminosity $155 L_{\odot}$. The distributions of the density and velocity are monotonic and are described well by the power laws $\rho \propto r^{-3 / 2}$ and $v \propto r^{-1 / 2}$, whose indices are characteristic for the self-similar solutions in the case of spherically symmetric accretion [7] (they are shown by the dash.dot line in Fig. 1). At the border of the sink cell, the hydrogen number density is close to $10^{8} \mathrm{~cm}^{-3}$, the accretion velocity has increased to $\approx 10 \mathrm{~km} / \mathrm{s}$. The temperature of the gas and dust in the inner regions is governed by the IR radiation of the protostar, while it continues to be determined by the interstellar radiation at the outer boundary of the cloud. The dash-dot line in Fig. 2 shows the distribution of the temperature of the stellar radiation in the absence of absorption, calculated using the expression $T(r)=\left(\frac{L_{\text {cell }}}{4 \pi a c}\right)^{1 / 4} r^{-1 / 2}$. The model distribution is located slightly above this dependence, due to the finite optical depth of the envelope and the additional heating due to adiabatic contraction.

\section{APPROXIMATE METHOD OF COMPU- TATION OF THERMAL EVOLUTION}

The mathematical model describing the thermal structure of the protostellar cloud is fairly complex. The direct realization of the method used to numerically solve the corresponding system of equations in the multi-dimensional case involves the solution of a number of additional problems of a technical nature. In particular, a highly sparse $M \times M$ matrix must be inverted, where $M$ is the total number of cells in the computational grid. The classical methods for solving such problems (the method of Gauss, LU decomposition, etc.) are very inefficient. Complex iterative algorithms are used, such as multi-grid methods, the methods of Krylov, etc. [24]. The final choice of the method used in the multi-dimensional case should be determined by the desired compromise between accuracy, computational time, and complexity of the realization of the method. The use of adequate approximate methods in the multi-dimensional calculations is an attractive idea. We devised an approximate method for the solution of the system of equations describing the thermal structure of the protostellar cloud, which we call the "adaptive $\tau$-approximation". In this method, the diffusion operator in the equation for the IR energy is replaced by the 
local (coordinate dependent) algebraic operator

We devised an approximate method for the solution of the system of equations describing the thermal structure of the protostellar cloud, which we call the "adaptive $\tau$-approximation". In this method, the diffusion operator in the equation for the IR energy is replaced by the local (coordinate dependent) algebraic operator

$$
\nabla \cdot\left(\frac{1}{\sigma_{\mathrm{R}}} \nabla E_{\mathrm{r}}\right)=-\frac{E_{\mathrm{r}}-E_{\mathrm{bg}}}{\tau} .
$$

Here, $E_{\mathrm{bg}}$ is the energy density of the background IR radiation and $\tau$ the characteristic diffusion time of the IR radiation. In essence, the adaptive $\tau$-approximation describes the release of IR radiation energy at a given point over a characteristic local timescale $\tau$, which is determined by the distribution of the radiation sources and the diffusion coefficient in the cloud. Generally speaking, various procedures can be used to calculate $\tau$ in the algebraic operator. We chose $\tau$ so that the stationary solution obtained using the $\tau$-approximation coincides with the exact solution.

The coefficient $\tau$ is computed as follows. Consider a stationary distribution of the gas and dust temperatures and the IR and UV energy. In this case, the subsystem of equations describing the thermal structure of the cloud can be reduced to the equation

$$
\nabla \cdot\left(\frac{1}{\sigma_{\mathrm{R}}} \nabla \bar{E}_{\mathrm{r}}\right)=Q
$$

where

$$
Q=\Lambda_{\mathrm{g}}-\Gamma_{\mathrm{g}}-S .
$$

Here, $\Lambda_{\mathrm{g}}$ is the gas cooling function via the emission of molecular line radiation, $\Gamma_{\mathrm{g}}$ the gas heating function via cosmic rays and photoelectrons, and $S$ the dust heating function via external UV radiation. A bar denotes the steady-state distribution of the energy density. Solving this equation taking into account of the necessary boundary conditions yields an approximate distribution for the energy density of the IR radiation. The coefficient $\tau$ can then be found using (20) and the corresponding approximate equation

$$
-\frac{\bar{E}_{\mathrm{r}}-E_{\mathrm{bg}}}{\tau}=Q .
$$

Technically, the adaptive $\tau$-approximation method can be implemented substantially more easily than the "full" method described above. Replacing the diffusion operator with an algebraic operator leads to a local nonlinear system of equations for determining $T_{\mathrm{g}}^{n+1}, T_{\mathrm{d}}^{n+1}$, and $T_{\mathrm{r}}^{n+1}$. In turn, the finite-difference equation (21) is linear $\left(\sigma_{\mathrm{R}}\right.$ and $Q$ depend on the previous time step) and its solution is appreciably simpler than the solution of the general system of equations.

Consider the spherically symmetric case, assuming that the energy density of the IR radiation is equal to the background energy density $E_{\mathrm{bg}}$ at the outer boundary of the cloud, at $r=R$. At the inner boundary of the cloud, $r=r_{1}$, the flux of IR radiation from the central protostar is specified to be $F=F_{\text {cell }}$. Integration of equations (21) and (23) yields

$$
\tau=\frac{1}{Q}\left\{\int_{r}^{R} d \xi \frac{\sigma_{\mathrm{R}}(\xi)}{\xi^{2}}\left[\int_{r_{1}}^{\xi} d \eta \eta^{2} Q(\eta)+r_{1}^{2} F_{\text {cell }}\right]\right\} .
$$


Note that the IR luminosity of the protostar is then $L_{\text {cell }}=4 \pi r_{1}{ }^{2} F_{\text {cell }}$. In the case when $\sigma_{\mathrm{R}}=$ const and $Q=$ const, we have

$$
\tau=\frac{r_{1}^{2} F_{\text {cell }}}{Q} \sigma_{\mathrm{R}}\left(\frac{1}{r}-\frac{1}{R}\right)+\frac{1}{3} \sigma_{\mathrm{R}}\left[\frac{R^{2}-r^{2}}{2}-r_{1}^{3}\left(\frac{1}{r}-\frac{1}{R}\right)\right] .
$$

For regions sufficiently far from the cloud boundary, we can assume $r_{1} \ll R$ and $r \ll R$. Therefore, we can obtain from (25) the approximate expression

$$
\tau \approx \frac{r_{1}^{2} F_{\text {cell }}}{Q} \frac{\sigma_{\mathrm{R}}}{r}+\frac{1}{6} \sigma_{\mathrm{R}} R^{2} .
$$

Thus, the possibility of taking into account the additional source of IR radiation due to the central protostar is an important advantage of the adaptive $\tau$-approximation method. This makes it possible to use this method to calculate the thermal structure of a protostellar cloud in the multi-dimensional case with the constructed model for the protostar.

The adaptive $\tau$-approximation method gives the exact solution (or close to it) in two important special cases. In the initial stages of the contraction of the protostellar cloud, the medium is optically thin to IR radiation. This means that the timescale $\tau$ is much shorter than the dynamical timescale, which can be estimated as the cloud free-fall timescale $t_{f f}$. As a result, the diffusion operator dominates, and we obtain nearly the steady-state energy density of the IR radiation, close to the background value $E_{\mathrm{bg}}$. Later, $\tau$ increases proportional to the density in the central regions of the cloud, while dynamical timescale decreases with increasing density proportional to $\rho^{-1 / 2}$. Therefore, in late stages of the contraction, the situation will become exactly the opposite: $\tau$ will be much longer than the dynamical timescale. In this case, the diffusion operator is not important, and the energy density of the IR radiation will vary mainly due to the source terms. Therefore, we expect that the $\tau$-approximation will yield a solution close to the solution obtained using the rigorous diffusion operator.

Figure 3 shows the distribution of the dust temperature $T_{\mathrm{d}}$ for various epochs in the evolution of a protostellar cloud obtained using the $\tau$-approximation (tau), Eddington approximation (edd), and diffusion approximation with a flux limiter (fld) (for a description of the Eddington method and the diffusion approximation with a flux limiter, see Section 2). The $\tau$-approximation and Eddington approximation agree fairly well with the "fld" method in the prestellar phase, up to the time of the formation of the hydrostatic core. Significant differences between the distributions are observed in the accretion phase. The decrease in the dust temperature at 100-1000 AU is stronger in the Eddington approximation than in the "FLD" approach. This is associated with the ill-posed nature of the Eddington approximation for the streaming regime. The temperature obtained is higher in the $\tau$-approximation than in the Eddington approximation, but lower than in the "FLD" approximation. Although the adaptive $\tau$-approximation yields a significant error, it reproduces the behavior of the temperature fairly well, and is suitable for simple (preliminary) calculations of the evolution of protostellar clouds, as well as calculations of the evolution of optically thick protostellar disks. 

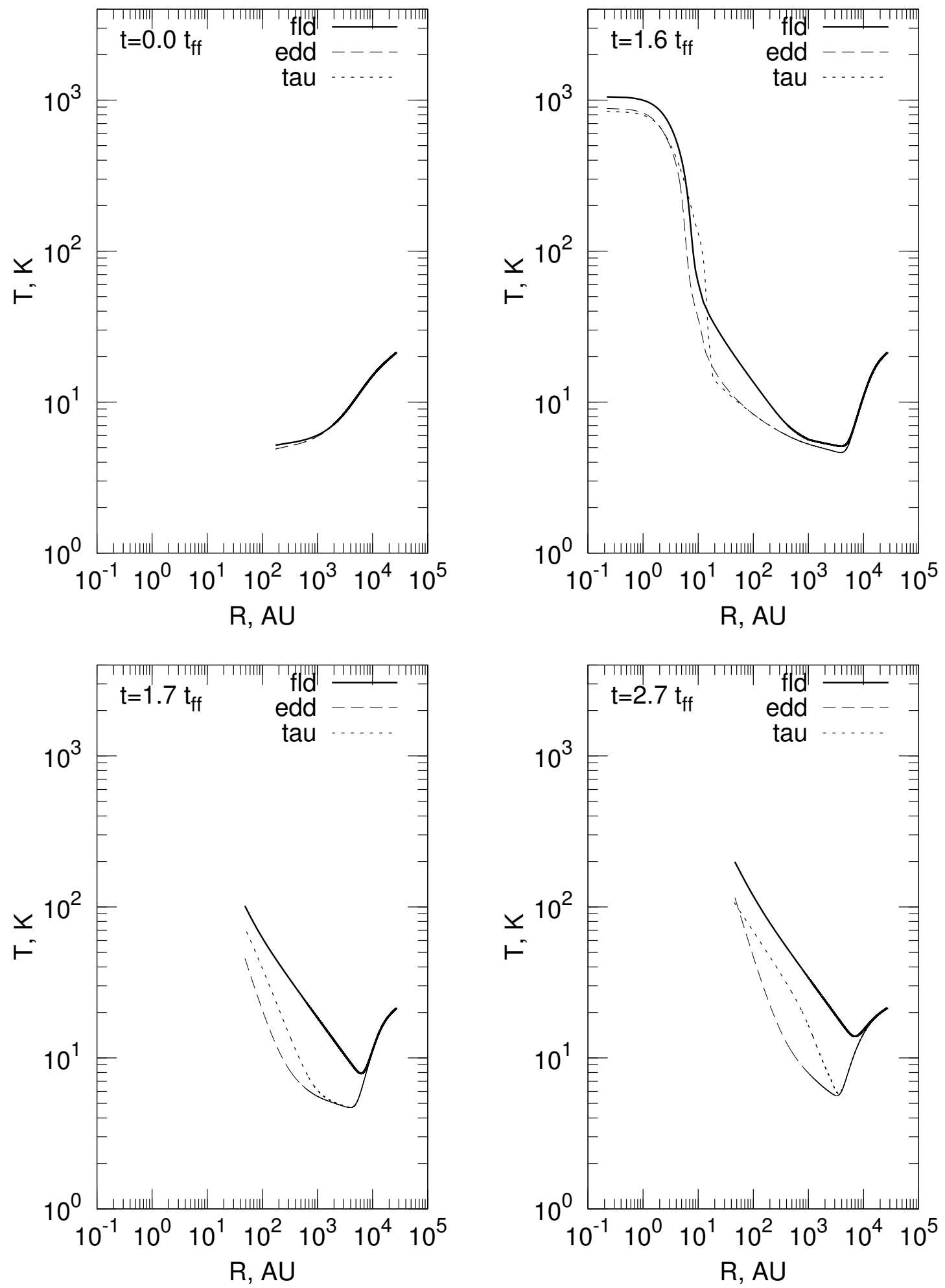

Figure 3: Distributions of the dust temperature $T_{\mathrm{d}}$ for various epochs during the evolution of a protostellar cloud, obtained using the $\tau$-approximation (tau), Eddington approximation (edd), and the diffusion approximation with a flux limiter (fld). 


\section{COMPARISON WITH OBSERVATIONS OF PRESTELLAR AND PROTOSTELLAR CORES}

The main aim of the thermal model presented here is a direct comparison of the results of the dynamical calculations with observations. The Herschel Space Observatory has obtained rich observational data on various protostellar objects in recent years. In particular, the spatial and thermal structure of a number of dense molecular clouds, including both prestellar and protostellar cores, were studied in [25-27]. The distributions of the surface number density of hydrogen $N_{\mathrm{H}}^{\mathrm{obs}}$ and the dust temperature averaged over the line of sight $\tilde{T}_{\mathrm{d}}^{\text {obs }}$ for a number of prestellar and protostellar cores are presented in [27]. It is natural for us is to attempt to reproduce these distributions using our model. Two circumstances must be taken into account when modeling the distributions of $N_{\mathrm{H}}$ and $\tilde{T}_{\mathrm{d}}$ based on the theoretical distributions $n_{\mathrm{H}}$ and $T_{\mathrm{d}}$. First, the temperature is not an additive quantity, and the averaging along the line of sight must be carried out consistently with the method used in [27] to reconstruct the distributions. Second, $N_{\mathrm{H}}^{\mathrm{obs}}$ and $\tilde{T}_{\mathrm{d}}^{\text {obs }}$ were determined from observations of distributions of the radiation intensity with finite angular resolution, $\mathrm{HPBW}=36^{\prime \prime}$.

Let us consider the radiation intensity $I_{\nu}$ in the optically thin approximation:

$$
I_{\nu}=m_{\mathrm{H}} \kappa_{\nu} \int_{0}^{N_{\mathrm{H}}} B_{\nu}\left(T_{\mathrm{d}}\right) d N,
$$

where $\kappa_{\nu}\left[\mathrm{cm}^{2} / \mathrm{g}\right]$ is the opacity per gram of gas, $m_{\mathrm{H}}$ the atomic mass of hydrogen, $N$ the surface number density of hydrogen along the line of sight, $N_{\mathrm{H}}$ the total surface number density of hydrogen, and $B_{\nu}$ the Planck function. In essence, it was assumed in [27] that

$$
I_{\nu}^{\mathrm{obs}}=m_{\mathrm{H}} \kappa_{\nu} B_{\nu}\left(\tilde{T}_{\mathrm{d}}^{\mathrm{obs}}\right) N_{\mathrm{H}}^{\mathrm{obs}} .
$$

In other words, distributions $\tilde{T}_{\mathrm{d}}^{\text {obs }}$ and $N_{\mathrm{H}}^{\mathrm{obs}}$ are found for which the spatial distribution (28) satisfactorily describes the observed maps at all available frequencies. Since $I_{\nu}^{\mathrm{obs}}$ is a convolution of the true observed radiation intensity with the telescope beam, the distribution. $\tilde{T}_{\mathrm{d}}^{\text {obs }} \mathrm{d}$ is a result of averaging not only along the line of sight, but also along the angular coordinate.

To construct the theoretical distribution $\tilde{T}_{\mathrm{d}}^{\text {conv }}$, we computed the distribution of the radiation intensity $I_{\nu}(x, y)$ using Eq. (27) and convolved it with the Gaussian telescope beam $W(x, y)$ :

$$
I_{\nu}^{\mathrm{conv}}(x, y)==\int W\left(x-x^{\prime}, y-y^{\prime}\right) I_{\nu}\left(x^{\prime}, y^{\prime}\right) d x^{\prime} d y^{\prime}
$$

In this formula, $W(x, y)=\frac{1}{\pi H^{2}} \exp \left(-\frac{x^{2}+y^{2}}{H^{2}}\right)$, where $H=\frac{\mathrm{HPBW}}{2 \sqrt{\ln 2}}$, and HPBW is the half-power beam width in radians. We compared $\tilde{T}_{\mathrm{d}}^{\text {obs }}$ with the temperature.$\tilde{T}_{\mathrm{d}}^{\text {conv }}$ found from the expression

$$
B_{\nu}\left(\tilde{T}_{\mathrm{d}}^{\text {conv }}\right)=\frac{I_{\nu}^{\text {conv }}}{m_{\mathrm{H}} \kappa_{\nu} N_{\mathrm{H}}^{\text {conv }}}
$$

Averaged over the telescope beam, the surface number density is 

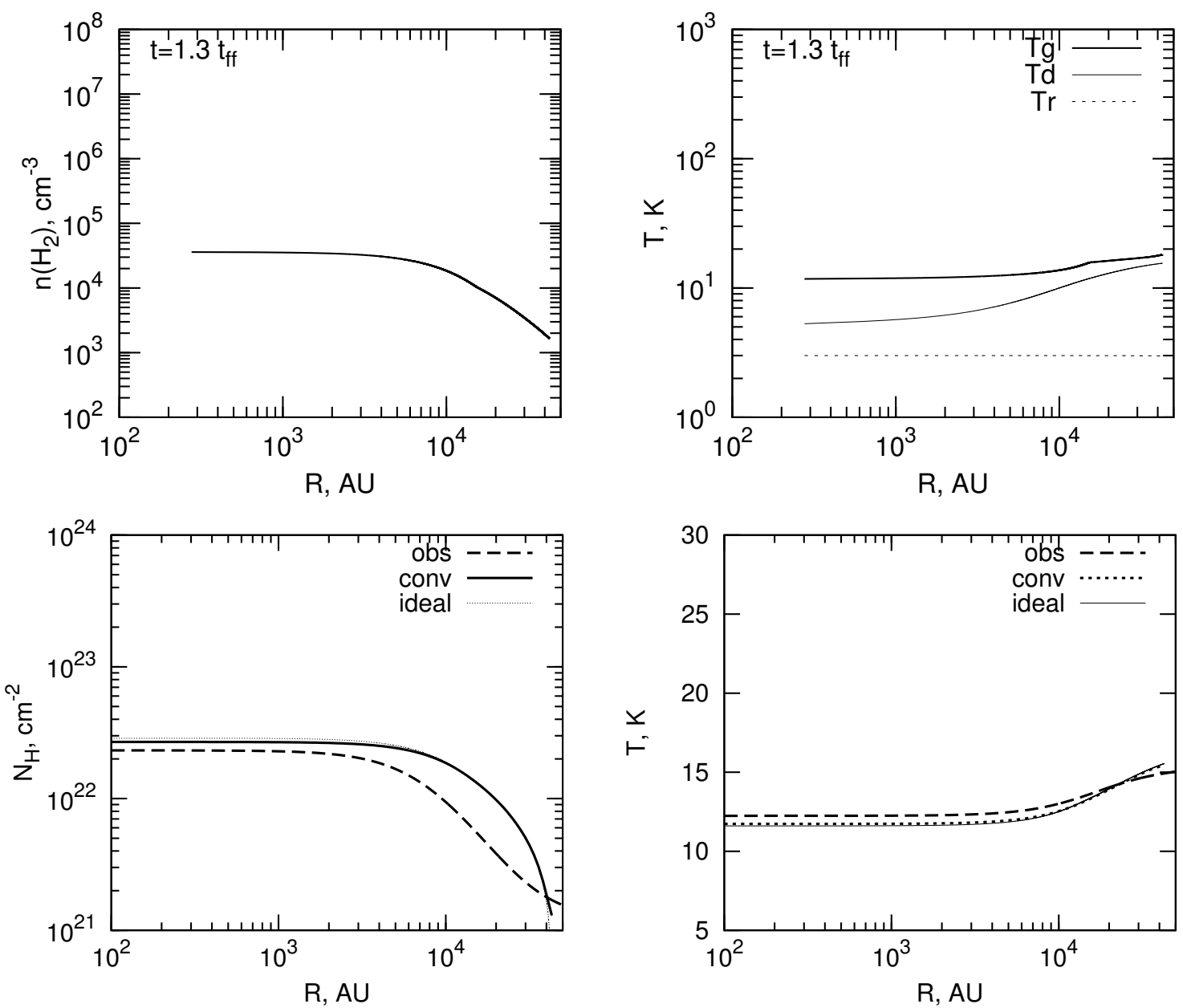

Figure 4: Distributions for the prestellar core: hydrogen number density (upper left); gas, dust, and radiation temperature (upper right); surface density (lower left); and dust temperature weighted over the line of sight (lower right). In the lower panels, the model distributions are shown by the solid curves and the observed distributions from [27] by the bold dashed curves. The model distributions were derived by convolving the ideal distributions (shown by the thin dashed curves) with a Gaussian beam with $\mathrm{HPBW}=36^{\prime \prime}$.

$$
N_{\mathrm{H}}^{\mathrm{conv}}==\int W\left(x-x^{\prime}, y-y^{\prime}\right) N_{\mathrm{H}}\left(x^{\prime}, y^{\prime}\right) d x^{\prime} d y^{\prime} .
$$

Note that the method for averaging the temperature described above does not require knowledge of the opacity $\kappa_{\nu}$ in $(27)$ and (30), since it cancels out in the final formula. However, the method does depend on the wavelength used in the Planck function. We used a wavelength of $160 \mathrm{mkm}$, close to the maximum of the observed radiation flux. We compared the model with the observed distributions averaged over the sample of sources shown in Fig. 8 of [27] by blue and red lines. When calculating the distributions $N_{\mathrm{H}}^{\text {conv }}$ and $\tilde{T}^{\text {conv }}$, we assumed that the cloud was located at a distance of $140 \mathrm{pc}$, and that the telescope beam was Gaussian with $\mathrm{HPBW}=36^{\prime \prime}$. 

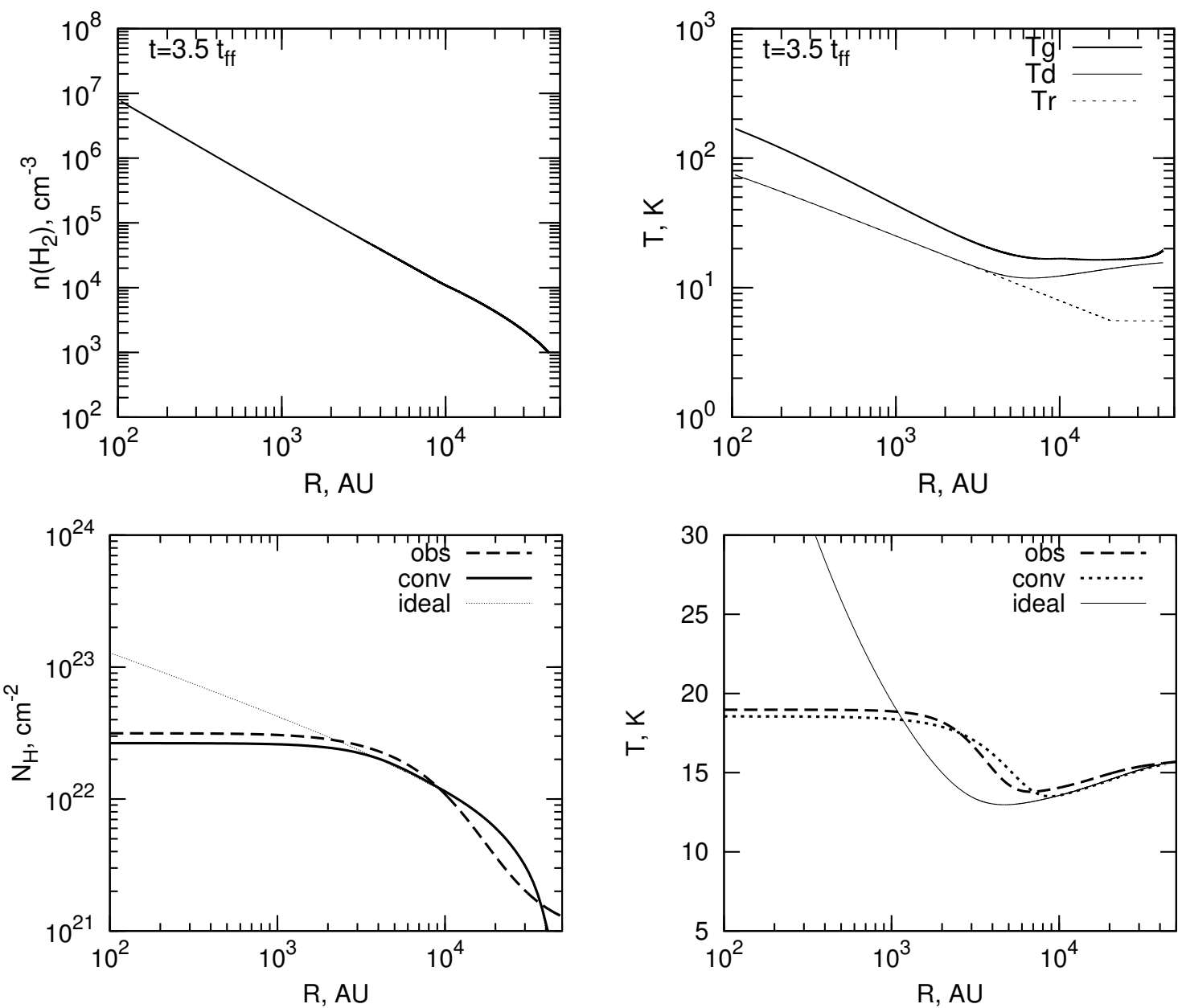

Figure 5: Same as in Fig. 4 for protostellar core.

Table 1: Parameters of the prestellar cloud model

\begin{tabular}{l|c}
\hline \multicolumn{1}{c|}{ Parameter } & Value \\
\hline Central concentration $\mathrm{H}_{2}$ & $4 \times 10^{4} \mathrm{~cm}^{-3}$ \\
Radius & $5 \times 10^{4} \mathrm{AU}$ \\
Mass & $6 M_{\odot}$ \\
Temperature of background UV radiation & $10^{4} \mathrm{~K}$ \\
Dilution of background UV radiation & $1.5 \times 10^{-15}$ \\
Evolutionary time span & $330 \mathrm{ka}$ \\
\hline
\end{tabular}

We suppose that the mean observed distributions of the density and temperature for the prestellar cores correspond to cores close to hydrostatic and thermal equilibrium. Thus, the main parameters of the model are the initial central density, the radius of the cloud, the parameters of the external radiation field, and the evolution time. We selected the model parameters for the prestellar cores so that the value $N_{\mathrm{H}}^{\text {conv }}=2 \times 10^{22} \mathrm{~cm}^{-2}$ in the direction toward the cloud center coincided with the observed value, and the cloud radius was equal to the maximum value in the 
distribution presented in [27], namely, $R=5 \times 10^{4}$ AU. The selected parameters of the external radiation field were such that $\tilde{T}_{\mathrm{d}}^{\text {conv }}=15 \mathrm{~K}$ at the cloud boundary. The evolution time for the model was $1.3 t_{\mathrm{tff}}$; during this time, the central density doubled compared to the initial state. Note that the model parameters were not rigorously varied, and therefore are not strictly optimal, as we did not aim to accurately reproduce the observational data. Our goal was to determine whether our model was consistent with the available observational data.

Figure 4 shows the distributions of the density and temperature in the model for the prestellar core, together with a comparison between the derived distributions $N_{\mathrm{H}}^{\text {conv }}$ and $\tilde{T}_{\mathrm{d}}^{\text {conv }}$ and the observed distributions. The model parameters are given in the table.

The results were obtained in the diffusion approximation with a flux limiter. The model and observed temperature distributions agree both qualitatively and quantitatively. The temperature is lower in the direction toward the center than in the direction toward the outer region of the cloud, as a consequence of external heating. At the same time, the surface density distributions differ significantly. The theoretical distribution has a more extended central plateau and steepe density decline toward the edge. At a distance of $2 \times 10^{4} \mathrm{AU}$, the model density is a factor of three higher than the observed value. One possible reason for this discrepancy in the observed and theoretical surface-density distributions is that the assumption that the prestellar cloud was initially in a quasi-equilibrium state was too crude. Indeed, according to modern gravo-turbulent concept of star formation [28], prestellar cores form in the turbulent interstellar medium. As a result of collisions of turbulent flows, gravitationally bound fragments form, whose density distributions may differ significantly from equilibrium distributions.

We suppose that the mean observed density and temperature distributions for the protostellar cores correspond to clouds that are a product of the evolution of the prestellar cores and are in a phase of developed accretion. Therefore, the main parameter of the model is the evolution time. We choose this time to be $t=$ $3.5 t_{\mathrm{ff}}$, when the observed central surface number density $N_{\mathrm{H}}^{\text {conv }}=3 \times 10^{22} \mathrm{~cm}^{-2}$ is reproduced. Figure 5 shows model distributions of the density and temperature at this time together with the corresponding distributions of $N_{\mathrm{H}}$ and $\tilde{T}$ and the observed profiles.

At this time, the age of the star in the model is $340000 \mathrm{yrs}$, its mass is $2.9 M_{\odot}$, the accretion rate is $6 \times 10^{-6} M_{\odot} / \mathrm{yr}$, the photospheric luminosity of the star is $23 L_{\odot}$, and the accretion luminosity is $45 L_{\odot}$. Overall, the theoretical and observed temperature distributions agree well. Note also that it is necessary to take the convolution into account when interpreting the observations, since the model temperature distribution unweighted by the telescope beam (thin solid curve) differs strongly from the convolved distribution. At the same time, small differences in the distributions of the surface number density are observed in the outer parts of the cloud. This is obviously related to the initial conditions, similar to the situation with the prestellar cores.

\section{CONCLUSION}

We have presented a modification of amodel developed earlier for the calculation of the thermal structure of a collapsing protostellar cloud. The modified model is 
intended for the calculation of the prestellar evolutionary stage of the cloud, as well as modeling of the accreting envelope after the formation of the protostar. The main improvements of the model are the introduction of the sink-cell formalism, calculation of the luminosity using a model for the evolution of the young star, and replacement of the Eddington approximation with the diffusion approximation with a flux limiter in the treatment of the radiative transfer.

We have applied the model to the evolution of a protostellar cloud, right up to the formation of the first hydrostatic core and the subsequent accretion phase. In the inner region of the accreting envelope $\left(r<10^{3}-10^{4} \mathrm{AU}\right)$, the temperature is governed by the radiation from the young star. In this zone, the temperatures of the gas and dust are equal and are well described by the distribution $T \propto r^{-1 / 2}$. The thermal structure of the outer layers of the envelope $\left(r>10^{3}-10^{4} \mathrm{AU}\right)$ is determined by interstellar radiation; the gas and dust temperatures rise toward the edge and may differ significantly.

We have used our model to explain the distributions of the surface density and temperature averaged over the line of sight derived fromobservations of low mass prestellar and protostellar cores of molecular clouds obtained with the Herschel Space Observatory. The model and observed temperature distributions are in good agreement, for both the prestellar and protostellar cores. At the same time, the distributions of the surface density display some differences, probably due to the incorrect assumption that the cloud was initially in a quasi-hydrostatic state. The model can be adapted for multi-dimensional calculations, and can also be used to calculate the chemical structure of protostellar objects.

The authors acknowledge Ralf Launhardt for providing observational data and B.M. Shustov for useful discussions.

\section{References}

[1] Zhilkin, A. G. and Pavlyuchenkov, Y. N. and Zamozdra, S. N., Astron. Rep., 53, 590 (2009).

[2] Ya. N. Pavlyuchenkov and A. G. Zhilkin, Astron. Rep., 57, 641 (2013).

[3] R. B. Larson, Monthly Not. Roy. astron. Soc., 145, 27 (1969)

[4] H. Masunaga, S.-I. Inutsuka, S.-i., Astrophys. J., 531, 350 (2000)

[5] N. Vaytet, G. Chabrier, E. Audit, et al., Astron. \& Astrophys., 557, 90 (2013)

[6] A. V. Tutukov and B.M. Shustov, Sov. Astron., 25, 61 (1981).

[7] F. H. Shu, Astrophys. J., 214, 488 (1977)

[8] Lou Y.-Q., Wang W.-G., Monthly Not. Roy. astron. Soc., 372, 885 (2006)

[9] P. Cassen, A. Moosman, Icarus, 48, 353 (1981)

[10] P. N. Foster, R. A. Chevalier, Astrophys. J., 416, 303 (1993)

[11] S. Ogino, K. Tomisaka, F. Nakamura, Pub. Astron. Soc. Pacific, 51, 637 (1999)

[12] E. Vorobyov, S. Basu, Monthly Not. Roy. astron. Soc., 360, 675

[13] H. Masunaga, S. M. Miyama, S. Inutsuka, Astrophys. J., 495, 346 (1998) 
[14] S. S. R. Offner, R. I. Klein, C. F. McKee, M. R. Krumholz, Astrophys. J., 703, $131(2009)$

[15] N. Vaytet, E. Audit, G. Chabrier, et al. Astron. \& Astrophys., 543, 60 (2012)

[16] M. M. Dunham, E. I. Vorobyov, Astrophys. J., 747, 52 (2012)

[17] K. Tomida, et al., Astrophys. J., 763, 29 (2013)

[18] C. Federrath, R. Banerjee, P. C. Clark, R. S. Klessen, 2010, Astrophys. J., 713, 269 (2010)

[19] G. Chabrier and I. Baraffe, Astron. and Astrophys., 327, 1039 (1997)

[20] I. Baraffe, G. Chabrier and J. Gallardo, Astrophys. J., 702, L27 (2009)

[21] I. Baraffe, E. I. Vorobyov, G. Chabrier, Astrophys. J., 756, 118 (2012)

[22] K. Tomida, M. N. Machida, K. Saigo, et al., Astrophys. J. Letters, 725, 329, (2010)

[23] C. D. Levermore, G. C. Pomraning, 1981, Astrophys. J., 248, 321 (1981)

[24] W. J. Rider, D. A. Knoll, G. L. Olson, J. Comp. Phys., 152, 164 (1999)

[25] A. Stutz, R. Launhardt, H. Linz, et al., Astron. \& Astrophys., 518, 87 (2010)

[26] M. Nielbock, R. Launhardt, J. Steinacker, et al., Astron. \& Astrophys., 547, $11(2012)$

[27] R. Launhardt, A.M. Stutz, A. Schmiedeke et al., Astron. \& Astrophys. 551, $98(2013)$

[28] M.-M. Mac Low, R. S. Klessen, Rev. Mod. Phys., 76, 125 (2004) 